\title{
The Seasonal Abundance and Distribution of the Pelagic Young of Teleostean Fishes Caught in the Ring-Trawl in Offshore Waters in the Ply- mouth Area.
}

\author{
By \\ F. S. Russell, D.S.C., B.A., \\ Naturalist at the Plymouth Laboratory.
}

With 2 Figures in the Text.

A LARGE number of collections were made with the 2-metre stramin ring-trawl in the years 1924, 1925 and 1926, to study the vertical distribution of young fish. Seeing that all these collections were made in exactly the same way, it was considered that they would form a good basis for a study of the quantitative differences in abundance of the different species at various times of the year. Accordingly, after 1926 the collections were supplemented in 1927, 1928 and 1929 by oblique hauls with the ring-trawl fishing at the same depths as those fished in the serial hauls in the study of the vertical distribution, that is the net was fished successively at the six different depths during half-an-hour's haul for 5 minutes at each depth. The results given in this report are all based on daylight catches.

In Table 3 are brought together the average monthly catches per half-hour's haul for each year for the post-larvæ of the various species. In the case of the 1924, 1925 and 1926 observations, which were based usually on six hauls each of ten-minutes' duration at six different depths, the total number of each species caught at all depths together at any one station is taken and from it the number caught per half-hour estimated. In the later years the oblique hauls were each of one half-hour's duration. In both cases these half-hour catches have been added together for each month in the year and divided by the number of catches per month. In this way an average monthly catch for each year was obtained (Table 3). By adding the averages for any one month and dividing by the number of years in which collections were made in that month, an average monthly catch was obtained for the period of six years covered by the researches (Table 1, p. 712). In Table 2 (p. 717) are given the dates in each year when collections were made, and it can be seen that although the winter months have been very poorly represented the important period of April to September has been fairly well covered.

Figure 1 shows the average monthly catches (as given in Table 1) for 


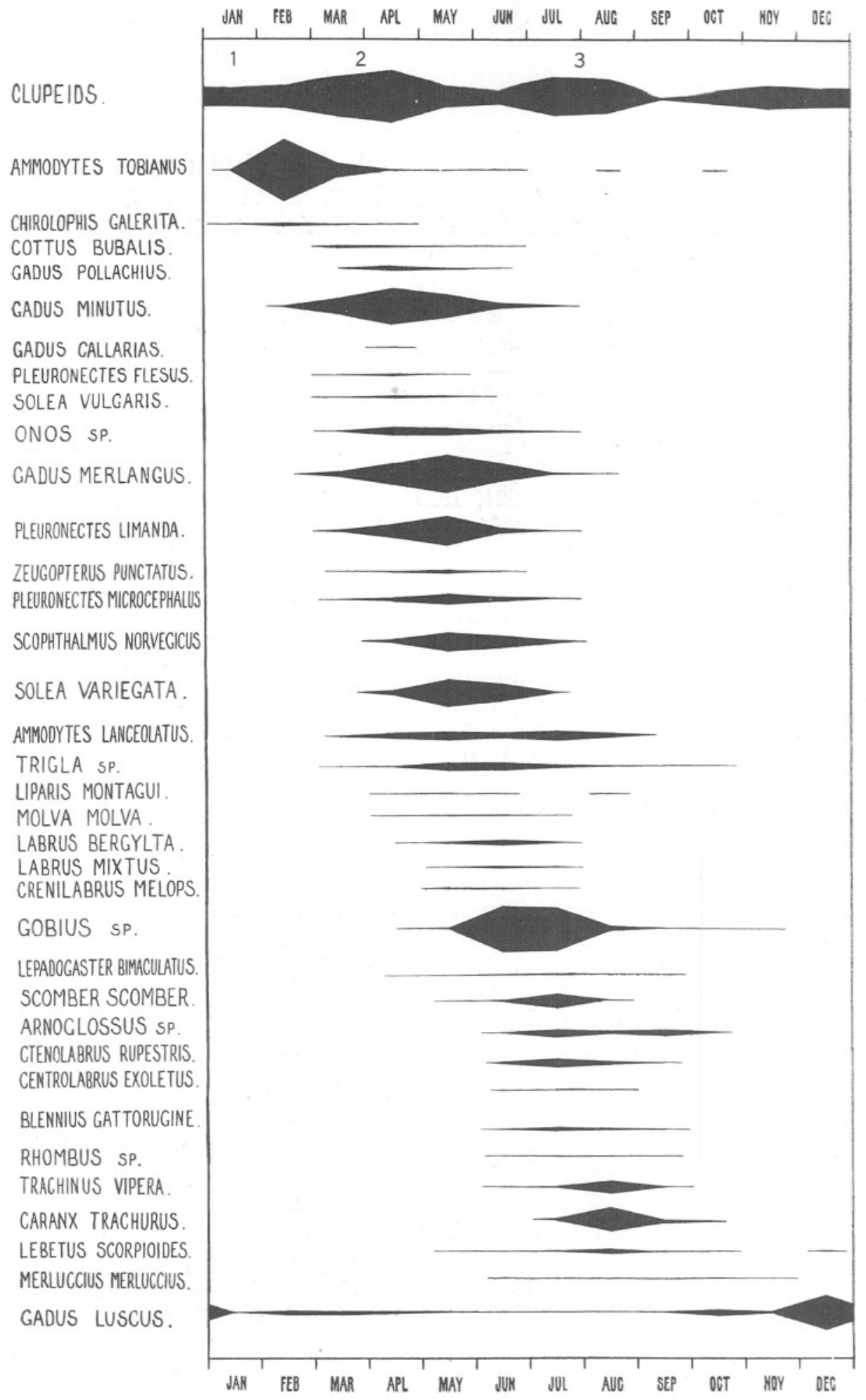

Frg. 1 (description opposite). 
each species in graphic form. The abundance curves for each species are drawn to the same scale so that from this figure one can see at a glance the comparative abundance of the post-larvæ of any species of fish at

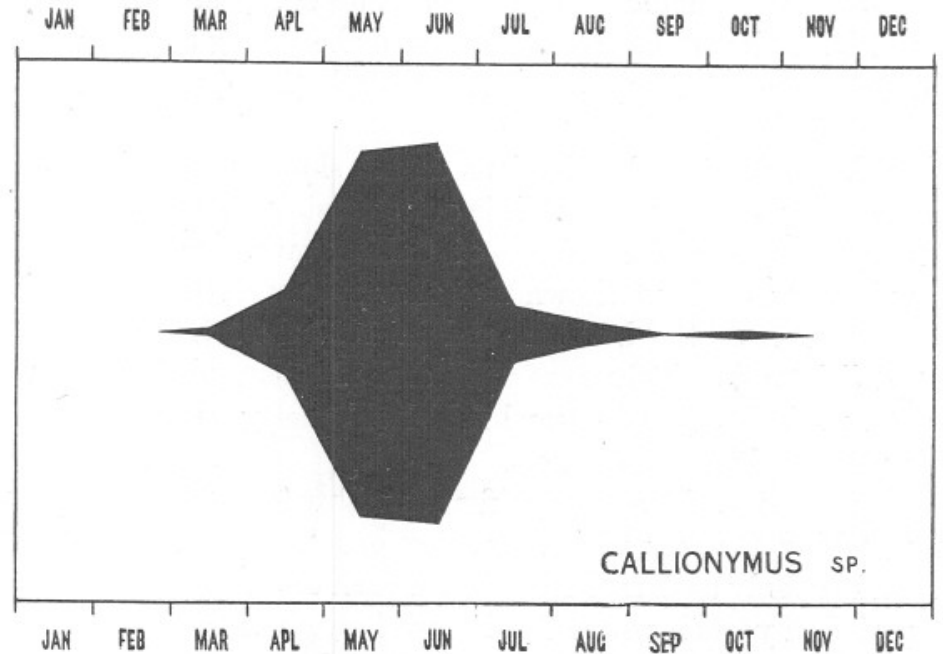

FIG. 2.-Diagram to show in graphic form the seasonal distribution and abundance of the post-larvæ of Callionymus sp., mostly $C$. lyra, in offshore waters off Plymouth. This figure is drawn to the same scale as Figure 1, and shows the marked superiority in the numbers of postlarvæ of this species over all other species, excepting perhaps the Gobies.

any time of the year. (This figure shows only those species whose average number for any month is one per half-hour's haul or more,)

In this publication only those observations made at the International Hydrographic Stations L4 and L6, or at the station two miles east of the Eddystone have been included, and from 1926 onwards practically all the collections were made from the latter position. The results are therefore indicative of the general offshore conditions. They should, if possible, be supplemented by a series of collections quite close inshore when possibly certain species, such perhaps as the wrasses, might appear more abundant.

\section{Description of Figure 1.}

Erg. 1.-Diagram to show in a graphic form the results expressed in Table 1. The blackened areas indicate the time of year at which post-larval stages of each species may be expected in the ring-trawl catches in offshore waters off Plymouth; each area is drawn to the same scale and indicates the comparative abundance of the postlarvæ of each species as averaged over the years 1924 to 1929 . Only those species whose post-larvæ appear with an average of over one specimen per half-hour's haul are shown here. In the case of the Clupeids the Figures 1, 2 and 3 indicate the periods when the catches may be expected to consist almost entirely of Herrings, Sprats, or Pilchards respectively.

N.B. The results for Gobies should probably be very much larger, these postlarva being generally near the bottom in the daytime and below the region sampled by the ring-trawl; the same also may hold for Gadus minutus.

NEW SERIES.-VOL. XVI. No. 3. MAY, 1930. 
A glance at Figure 1 shows how few species compared with the large number occurring in the area can be considered as abundant. The outstanding feature as shown in Figure 2 (drawn to the same scale as Figure 1) is the abundance of Callionymus sp. post-larvae probably mostly C. lyra. Ever since researches into the young stages of our fish were first systematically undertaken by Clark (1910) the abundance of this species has been noticeable.

Although the ring-trawl collections do not show the post-larval gobies to have been especially numerous it is quite possible that they are actually even more abundant than those of Callionymus. Catches with the bottom plankton net have shown that they are present in great numbers in the daytime near the bottom, and in 1927 the average catches for June and July respectively were 1354 and 3247 as against $2 \cdot 1$ and $2 \cdot 1$ in collections made by the ring-trawl fished obliquely ; this is many times more numerous than Callionymus.

It is thought that these average figures will form a good basis to work on in watching for any violent fluctuations in the future. They are presented here only as data, because until such observations have covered a long period of years it will not be known how great the differences from year to year must be before they can be regarded as significant.

There is an indication that the post-larval stages of certain spring spawners were but poorly represented in the year 1929, as for example, Gadus minutus, Pleuronectes microcephalus, Solea variegata, and Callionymus sp. Such differences will naturally tend to lower the averages given in Table 1. Similarly the year 1926 seems to have been marked by a rather unusual abundance of young mackerel, Scomber scomber; never in all the past records given by Clark (1914 and 1920) and Allen (1917) have so many been caught. This may perhaps have meant an easterly extension of their normal spawning-grounds, the abundance of the copepod, Calanus finmarchicus, being very great in this region that summer and hence perhaps the adults moving more in this direction. At any rate there is no indication from commercial catches that 1926 was an exceptionally good survival year, as they should probably have been appearing in the catches by now (1929).

The above instances have been cited to emphasize that we do not yet know what differences to look for as being significant. The results of the research on the vertical distribution of the post-larval fishes have shown that for the majority of species oblique daytime hauls with the net fished as deep as possible should give a fair picture of the quantities of young fish present (Russell, 1928, p. 833). In the case of post-larval Clupeids, however, these results do not give a correct impression (Russell, 1930 p. 649), although if they were unusually abundant any one year their increased numbers would perhaps show up in the daytime as well as at 
night. In the case of the Gobies, and also possibly Gadus minutus (Russell, 1930 , p. 650), the hauls probably have not gone deep enough to sample the zone of their maximum abundance.

A comparison of these results with the figures given by Allen (1917) shows that there have not been any marked changes in the composition of the catches since that date, and his averages for the years up to 1914 agree well with those given here. As regards the sizes of the post-larvæ caught, there will be some slight difference for each species as the season advances. When first any species starts to appear in the catches it is natural that the majority should be very young forms, and while a few of the smallest sizes are usually to be taken throughout the season there will be a gradual increase in size of the majority as time goes on, until in the last catches in which they appear the post-larvæ will mostly be in the neighbourhood of the size at which they disappear from the ring-trawl catches either owing to their leaving the plankton or having become strong enough swimmers to evade the net. In Table 3 are given for the commoner species the sizes within which the majority of post-larvæ taken in the ring-trawl lie ; larger sizes will of course be caught at times in the case of all species.

A bibliography is given here including all those papers that have been published dealing with the post-larval stages of Teleostean fishes in the Plymouth area (see pp. 720-722).

The months in which the maximum abundance of the post-larvæ of each species may be expected are given below.

MONTHS OF AVERAGE MAXIMAL ABUNDANCE OF POST-LARVA.

\begin{tabular}{|c|c|c|c|}
\hline JANUARY. & $\begin{array}{l}\quad \text { Febroary. } \\
\text { C. harengus } \\
\text { A. tobianus } \\
\text { C. galerita }\end{array}$ & $\begin{array}{l}\text { MaRch. } \\
\text { C. bubalis } \\
\text { A. cataphractus }\end{array}$ & $\begin{array}{l}\quad \text { APRIL. } \\
\text { C. sprattus } \\
\text { G. pollachius } \\
\text { G. minutus } \\
\text { G. callarias } \\
\text { P. flesus } \\
\text { S. vulgaris } \\
\text { Onos sp. }\end{array}$ \\
\hline $\begin{array}{l}\text { MaY. } \\
\text { merlangus } \\
\text { limanda } \\
\text { punctatus } \\
\text { microcephalus } \\
\text { norvegicus } \\
\text { variegata } \\
\text { rigla sp. } \\
\text { montagui }\end{array}$ & $\begin{array}{l}\quad \text { JuNE. } \\
\text { M. molva } \\
\text { Callionymus sp. } \\
\text { L. bergylta } \\
\text { L. mixtus } \\
\text { C. melops } \\
\text { Gobius sp. }\end{array}$ & $\begin{array}{l}\quad \text { JoLy. } \\
\text { C. pilchardus } \\
\text { R. raninus } \\
\text { Capros aper } \\
\text { S. scomber } \\
\text { Arnoglossus sp. } \\
\text { S. lascaris } \\
\text { S. lutea } \\
\text { A. lanceolatus } \\
\text { C. rupestris } \\
\text { C. exoletus } \\
\text { B. pholis } \\
\text { B. gattorugine } \\
\text { L. piscatorius } \\
\text { L. bimaculatus }\end{array}$ & $\begin{array}{l}\quad \text { AUgust. } \\
\text { Zeus faber } \\
\text { Rhombus sp. } \\
\text { S. cabrilla } \\
\text { T. vipera } \\
\text { C. } \text { trachurus } \\
\text { M. surmuletus } \\
\text { C. rubescens } \\
\text { L. scorpioides } \\
\text { B. ocellaris }\end{array}$ \\
\hline
\end{tabular}

September.

October.

November.

December.

M. merluccus

G. luscus 
TABLE 1.

Average Monthly Catches of Post-larve per Half-hour Haul with 2-metre Ring-trawl Fishing at all Depths, 1924-1929.

Clupeid sp.*

Gadus pollachius

G. merlangus

G. minutusं

G. luscus

G. callarias

Onos sp.

Molva molva

Merluccius merluccius

Raniceps raninus

Capros aper

Zeus faber

Arnoglossus sp.

Rhombus sp.

Scophthalmus norvegicus

S. unimaculatus

Zeugopterus punctatus

Pleuronectes limanda

P. flesus

P. microcephalus

Solea vulgaris

S. variegata

S. lascaris

Serranus cabrilla

Caranx trachurus

Mullus surmuletus

Ammodytes tobianus

A. lanceolatus

Cepola rubescens

$\begin{array}{lccccccccccc}\text { Jan. } & \text { Feb. } & \text { March. } & \text { April. } & \text { May. } & \text { June. } & \text { July. } & \text { Aug. } & \text { Sept. } & \text { Oct. } & \text { Nov. } & \text { Dec. } \\ 23 \cdot 7 & 30 \cdot 3 & 51 \cdot 6 & 70 \cdot 7 & 25 \cdot 0 & 15 \cdot 5 & 48 \cdot 4 & 42 \cdot 6 & 1 \cdot 6 & 16 \cdot 6 & 30 \cdot 0 & 22 \cdot 5 \\ - & - & 1 \cdot 2 & 7 \cdot 7 & 1 \cdot 6 & 0 \cdot 1 & - & - & - & - & - & - \\ - & - & 7 \cdot 8 & 32 \cdot 0 & 55 \cdot 9 & 32 \cdot 8 & 2 \cdot 3 & 0 \cdot 2 & - & - & - & - \\ - & 3 \cdot 9 & 24 \cdot 0 & 52 \cdot 5 & 33 \cdot 6 & 8 \cdot 8 & 0 \cdot 9 & - & - & - & - & - \\ 0 \cdot 9 & 6 \cdot 9 & 5 \cdot 4 & 3 \cdot 8 & 1 \cdot 8 & 0 \cdot 8 & 0 \cdot 1 & 1 \cdot 4 & 0 \cdot 7 & 7 \cdot 1 & 3 \cdot 9 & 49 \cdot 5 \\ - & - & - & 1 \cdot 1 & - & - & - & - & - & - & - & - \\ - & - & 0 \cdot 6 & 14 \cdot 8 & 10 \cdot 4 & 3 \cdot 3 & 0 \cdot 8 & - & - & - & - & - \\ - & - & - & 0 \cdot 1 & 3 \cdot 5 & 4 \cdot 6 & 0 \cdot 1 & - & - & - & - & - \\ - & - & - & - & - & 0 \cdot 3 & 0 \cdot 2 & 0 \cdot 2 & 0 \cdot 2 & 1 \cdot 6 & 0 \cdot 9 & - \\ - & - & - & - & - & 0 \cdot 04 & 0 \cdot 2 & 0 \cdot 2 & - & 0 \cdot 1 & - & - \\ - & - & - & - & - & 0 \cdot 04 & 0 \cdot 3 & 0 \cdot 3 & 0 \cdot 1 & - & - & - \\ - & - & - & - & - & - & - & 0 \cdot 1 & - & - & - & - \\ - & - & - & - & - & 0 \cdot 2 & 12 \cdot 4 & 7 \cdot 6 & 10 \cdot 5 & 2 \cdot 0 & - & - \\ - & - & - & - & - & 0 \cdot 2 & 1 \cdot 8 & 2 \cdot 4 & 0 \cdot 6 & - & - & - \\ - & - & - & 5 \cdot 8 & 29 \cdot 8 & 18 \cdot 3 & 6 \cdot 4 & - & - & - & - & - \\ - & - & - & - & - & + & + & - & - & - & - & - \\ - & - & 0 \cdot 6 & 1 \cdot 2 & 6 \cdot 2 & 0 \cdot 8 & - & - & - & - & - & - \\ - & - & 6 \cdot 6 & 21 \cdot 8 & 45 \cdot 3 & 8 \cdot 8 & 0 \cdot 2 & - & - & - & - & - \\ - & - & 0 \cdot 3 & 4 \cdot 5 & 0 \cdot 7 & - & - & - & - & - & - & - \\ - & - & 0 \cdot 3 & 6 \cdot 3 & 16 \cdot 1 & 8 \cdot 4 & 1 \cdot 5 & - & - & - & - & - \\ - & - & 0 \cdot 3 & 4 \cdot 9 & 1 \cdot 0 & 0 \cdot 04 & - & - & - & - & - & - \\ - & - & - & 5 \cdot 8 & 40 \cdot 5 & 29 \cdot 1 & 3 \cdot 4 & - & - & - & - & - \\ - & - & - & - & - & - & 0 \cdot 1 & - & - & 0 \cdot 4 & - & - \\ - & - & - & - & - & - & 0 \cdot 1 & - & - & - & - & - \\ - & - & - & - & - & - & - & 0 \cdot 3 & 0 \cdot 1 & 0 \cdot 2 & - & - \\ - & - & - & - & - & - & 3 \cdot 9 & 34 \cdot 4 & 3 \cdot 6 & 2 \cdot 0 & - & - \\ - & - & - & - & - & - & - & 0 \cdot 1 & - & - & - & - \\ 0 \cdot 6 & 90 \cdot 9 & 20 \cdot 7 & 2 \cdot 6 & 0 \cdot 1 & 0 \cdot 2 & - & 0 \cdot 2 & 0 \cdot 4 & 0 \cdot 2 & - & - \\ - & - & 2 \cdot 4 & 8 \cdot 6 & 13 \cdot 9 & 9 \cdot 1 & 14 \cdot 8 & 8 \cdot 1 & - & - & - & - \\ - & - & - & - & - & 0 \cdot 04 & 0 \cdot 1 & 0 \cdot 4 & - & - & - & \\ & - & & & & & & & & & & -\end{array}$


Callionymus sp.

Labrus bergylta

Ctenolabrus rupestris

Crenilabrus melops

Centrolabrus exoletus

Trachinus vipera

Trachinus draco

Scomber scomber

Gobius sp.\$

Lebetus scorpioides

Blennius ocellaris

B. pholis

B. gattorugine

Chirolophis galerita

Trigla sp.

Cottus bubalis

Agonus cataphractus

Liparis montagui

Lepadogaster bimaculatus

Lophius piscatorius

Belone vulgaris +

$\begin{array}{llll}- & - & 8 \cdot 7 & 89 \cdot 0 \\ - & - & - & 0 \cdot 1 \\ - & - & - & - \\ - & - & - & - \\ - & - & - & - \\ - & - & - & - \\ - & - & - & - \\ - & - & - & - \\ - & - & - & - \\ - & - & - & 0 \cdot 9 \\ - & - & - & - \\ - & - & - & - \\ - & - & - & - \\ - & - & - & - \\ 0 \cdot 3 & 3 \cdot 0 & 2 \cdot 0 & 0 \cdot 3 \\ - & - & 1 \cdot 5 & 3 \cdot 0 \\ - & - & 4 \cdot 5 & 2 \cdot 6 \\ - & - & 0 \cdot 3 & 0 \cdot 1 \\ - & - & - & 0 \cdot 3 \\ - & - & - & 0 \cdot 3 \\ - & - & 0 \cdot 3 & 0 \cdot 04 \\ - & - & - & -\end{array}$

$\begin{array}{cc}377 \cdot 6 & 39 \\ 1 \cdot 3 & \\ 0 \cdot 2 & \\ - & \\ - & \\ - & \\ - & \\ - & \\ 0 \cdot 1 & \\ 1 \cdot 8 & 68 \\ 0 \cdot 2 & \\ - & \\ 0 \cdot 1 & \\ - & \\ - & \\ 13 \cdot 4 & 1 \\ 0 \cdot 7 & \\ 0 \cdot 1 & \\ 1 \cdot 1 & \\ 0 \cdot 1 & \\ - & \\ - & \end{array}$

$\begin{array}{rr}95 \cdot 0 & 59 \cdot 2 \\ 9 \cdot 1 & 1 \cdot 2 \\ 5 \cdot 9 & 1 \cdot 4 \\ 2 \cdot 5 & 12 \cdot 0 \\ 2 \cdot 9 & 2 \cdot 5 \\ 1 \cdot 3 & 1 \cdot 9 \\ 0 \cdot 4 & 2 \cdot 6 \\ - & - \\ 5 \cdot 8 & 22 \cdot 8 \\ 68 \cdot 8 & 62 \cdot 9 \\ 0 \cdot 7 & 2 \cdot 7 \\ 0 \cdot 2 & 0 \cdot 4 \\ 0 \cdot 8 & 0 \cdot 8 \\ 2 \cdot 5 & 6 \cdot 4 \\ \overline{12 \cdot 5} & - \\ 0 \cdot 5 & - \\ \overline{0} & - \\ 2 \cdot 8 & - \\ 0 \cdot 3 & 1 \cdot 6 \\ - & 0 \cdot 8\end{array}$

$24 \cdot 1$
-
$\overline{4} \cdot 9$
$0 \cdot 7$
$0 \cdot 8$
$18 \cdot 3$
$\overline{0} \cdot 2$
$8 \cdot 0$
$8 \cdot 7$
$0 \cdot 8$
$0 \cdot 2$
$5 \cdot 2$
$\overline{3} \cdot 5$
-
$\overline{0}$
$0 \cdot 3$
$1 \cdot 3$
$0 \cdot 1$

\begin{tabular}{llll}
$1 \cdot 1$ & $9 \cdot 6$ & $0 \cdot 9$ & - \\
- & - & - & - \\
$\overline{0} \cdot 1$ & - & - & - \\
- & - & - & - \\
$\overline{1}$ & - & - & - \\
$0 \cdot 0$ & - & - & - \\
$\overline{1}$ & - & - & - \\
$1 \cdot 2$ & - & - & - \\
$1 \cdot 5$ & $0 \cdot 5$ & - & - \\
- & - & - & - \\
$\overline{0}$ & - & - & - \\
- & - & - & - \\
$\overline{0} \cdot 7$ & - & - & - \\
- & $0 \cdot 9$ & - & - \\
- & - & - & - \\
$\overline{0}$ & - & - & - \\
$\overline{0} \cdot 3$ & - & - & - \\
$0 \cdot 1$ & - & - & - \\
\hline
\end{tabular}

* Includes Clupea harengus, Clupea sprattus, and Sardina pilchardus. Post-larval herring occur mostly in January, February, and March ; sprat in March, April, and May, the increase being generally due to larger numbers of recently hatched sprat: from May till end of year the catches are mostly pilchard which spawn intermittently throughout the summer.

$\dagger$ Gadus minutus post-larvæ live normally very deep in the water in the daytime, and possibly these averages should be considerably higher to give a true picture.

* In calculating these averages for Scomber scomber, the mackerel, the results for 1926 have not been included as it is thought that perhaps this may have been an abnormal year (see Table 3 ).

$\S$ The post-larval gobies live very near the bottom in the daytime, and these averages should probably be very much higher.

- Consist of Trigla gurnardus, T. cuculus, and T. hirundo chiefly,

+ Less than $0 \cdot 1$ 
TABLE 2.

Dates on Which Collections were made.

\begin{tabular}{|c|c|c|c|c|c|c|c|}
\hline January & 1924 & 1925 & 1926 & 1927 & $\begin{array}{r}1928 \\
9 \text { th } \\
16 \text { th } \\
26 \text { th } \\
30 \text { th }\end{array}$ & 1929 & $\begin{array}{l}\text { Total } \\
\text { days. }\end{array}$ \\
\hline February & - & - & - & - & $\begin{array}{l}2 \text { nd } \\
20 \text { th } \\
27 \text { th }\end{array}$ & - & 3 \\
\hline March & - & - & - & - & $\begin{array}{r}5 \text { th } \\
21 \text { st } \\
30 \text { th }\end{array}$ & - & 3 \\
\hline April & - & $\left\{\begin{array}{r}2 \text { nd } \\
8 \text { th } \\
29 \text { th }\end{array}\right.$ & $\begin{array}{l}9 \text { th } \\
13 \text { th (i) } \\
13 \text { th (ii) } \\
22 \text { nd } \\
26 \text { th }\end{array}$ & $\begin{array}{l}4 \text { th } \\
14 \text { th } \\
26 \text { th }\end{array}$ & $\begin{array}{l}4 \text { th } \\
11 \text { th } \\
12 \text { th } \\
23 \mathrm{rd}\end{array}$ & $\left\{\begin{array}{l}10 \text { th } \\
19 \text { th } \\
23 \mathrm{rd} \\
29 \text { th }\end{array}\right.$ & 19 \\
\hline May & 29 th & $\begin{array}{l}19 \operatorname{th}(\mathrm{i}) \\
19 \operatorname{th}(\mathrm{ii})\end{array}$ & $\begin{array}{l}6 \text { th } \\
19 \text { th }\end{array}$ & $\begin{array}{l}2 \text { nd } \\
9 \text { th } \\
16 \text { th } \\
25 \text { th }\end{array}$ & - & $\left\{\begin{array}{r}6 \text { th } \\
13 \text { th } \\
23 \text { rd } \\
27 \text { th }\end{array}\right.$ & 13 \\
\hline June & 25th & $\begin{array}{l}4 \text { th (i) } \\
4 \text { th (ii) } \\
17 \text { th } \\
18 \text { th } \\
19 \text { th }\end{array}$ & $\begin{array}{l}3 \text { rd } \\
4 \text { th } \\
25 \text { th } \\
30 \text { th }\end{array}$ & $\begin{array}{l}\text { 2nd } \\
9 \text { th } \\
29 \text { th }\end{array}$ & - & $\left\{\begin{array}{r}6 \text { th } \\
11 \text { th } \\
25 \text { th }\end{array}\right.$ & 16 \\
\hline July & $\begin{array}{l}\text { lst } \\
15 \text { th } \\
16 \text { th }\end{array}$ & $\begin{array}{l}\text { lst (i) } \\
\text { lst (ii) } \\
16 \text { th } \\
29 \text { th }\end{array}$ & $\begin{array}{r}6 \text { th } \\
13 \text { th } \\
26 \text { th }\end{array}$ & $\begin{array}{l}8 \text { th } \\
12 \text { th } \\
21 \text { st } \\
26 \text { th }\end{array}$ & - & $\left\{\begin{array}{r}3 \mathrm{rd} \\
9 \mathrm{th} \\
18 \mathrm{th} \\
23 \mathrm{rd} \\
30 \mathrm{th}\end{array}\right.$ & 19 \\
\hline August & - & 6 th & 4 th & $\begin{array}{l}4 \text { th } \\
8 \text { th } \\
19 \text { th } \\
26 \text { th } \\
31 \text { st }\end{array}$ & - & $\left\{\begin{array}{c}9 \text { th } \\
15 \text { th } \\
22 \text { nd } \\
26 \text { th }\end{array}\right.$ & 11 \\
\hline September & - & - & 22 nd & $\begin{array}{l}6 \text { th } \\
15 \text { th } \\
19 \text { th }\end{array}$ & - & $\left\{\begin{array}{r}4 \text { th } \\
6 \text { th } \\
10 \text { th } \\
17 \text { th } \\
24 \text { th }\end{array}\right.$ & 9 \\
\hline October & - & - & - & $\begin{array}{l}4 \text { th } \\
13 \text { th } \\
18 \text { th } \\
24 \text { th }\end{array}$ & - & $\left\{\begin{array}{l}3 \text { rd } \\
10 \text { th } \\
16 \text { th }\end{array}\right.$ & 7 \\
\hline November & - & - & - & lst & - & - & 1 \\
\hline December & - & - & - & $\begin{array}{l}15 \text { th } \\
21 \text { st }\end{array}$ & - & - & 2 \\
\hline
\end{tabular}


TABLE 3 .

Average Monthly Catch of Post-larva per 30-minute Haul with RING-TRAWL.

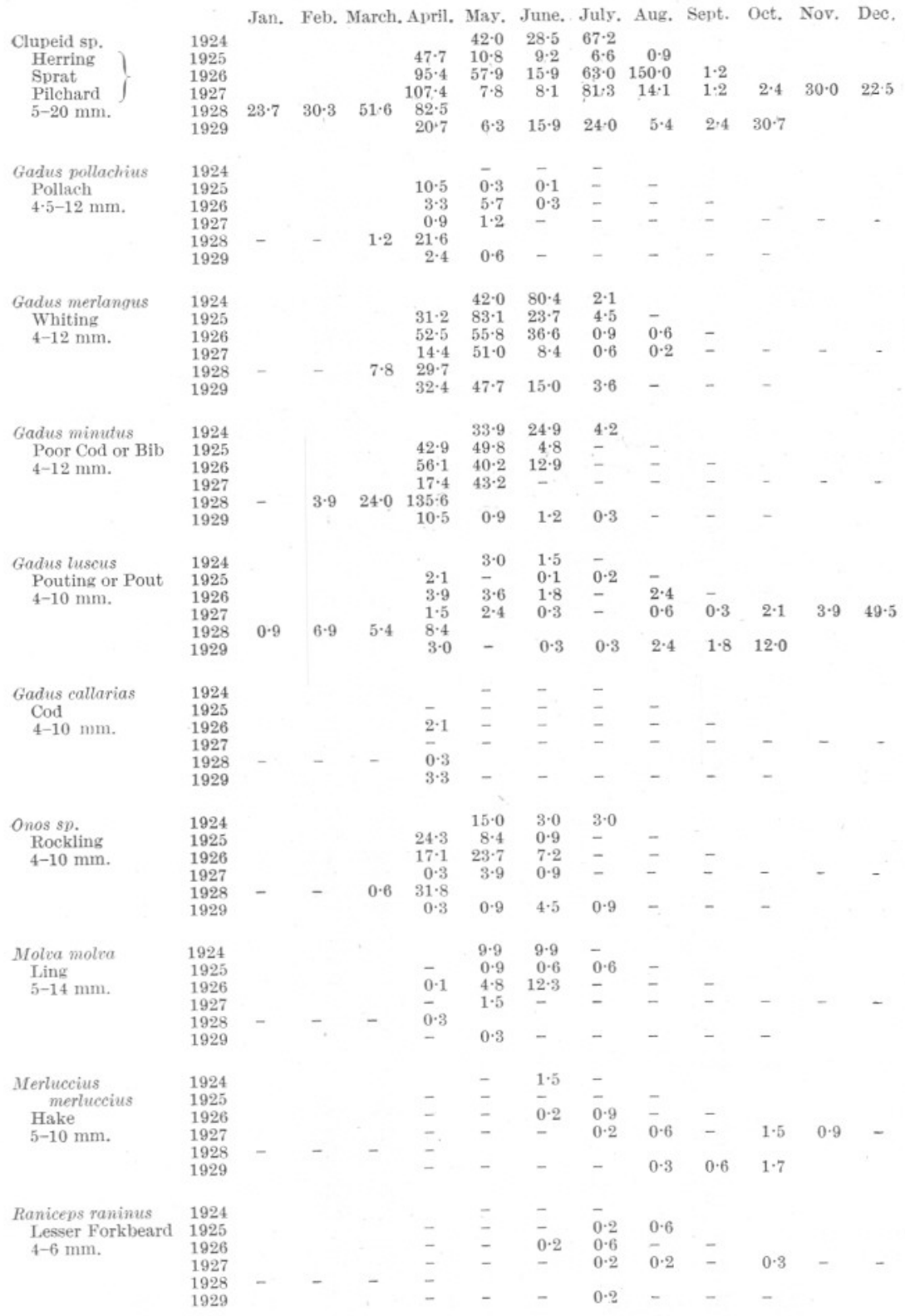




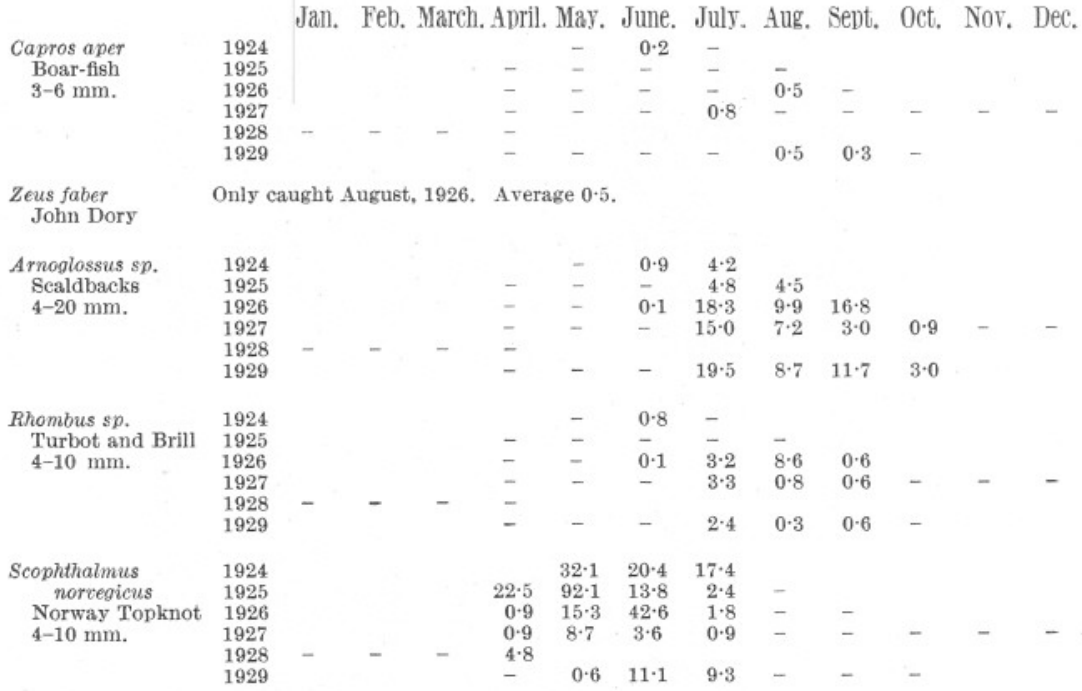

S. unimaculatus Only 6 eaught during the 6 years.

E1 $17 / 6 / 24,2 ; A, 1 / 7 / 25,1 ; 7 / 5 / 24,1 ; 17-18 / 7 / 25,2$ (dusk and dark).

\begin{tabular}{|c|c|c|c|c|c|c|c|c|c|c|c|c|c|}
\hline Zeugopterus & 1924 & & & & & $11 \cdot 1$ & - & - & & & & & \\
\hline punctatus & 1925 & & & & $1 \cdot 8$ & $12 \cdot 6$ & 0.2 & - & - & & & & \\
\hline Topknot & 1926 & & & & $0 \cdot 9$ & $2 \cdot 7$ & $3 \cdot 6$ & - & - & - & & & \\
\hline 4-10 $\mathrm{mm}$ & 1927 & & - & & $\begin{array}{l}1 \cdot 2 \\
0 \cdot 1\end{array}$ & $4 \cdot 2$ & - & - & - & - & - & - & - \\
\hline & $\begin{array}{l}1928 \\
1929\end{array}$ & - & - & $0 \cdot 6$ & $\begin{array}{l}2 \cdot 1 \\
-\end{array}$ & 0.6 & - & - & - & - & - & & \\
\hline Pleuronectes & 1924 & & & & & $24 \cdot 0$ & $15 \cdot 9$ & 0.9 & & & & & \\
\hline limanda & 1925 & & & & $26 \cdot 4$ & $69 \cdot 6$ & 3.9 & $0 \cdot 3$ & -. & & & & \\
\hline $\mathrm{Dab}$ & 1926 & & & & $24 \cdot 3$ & $50 \cdot 1$ & $9 \cdot 3$ & - & - & - & & & \\
\hline 5-12 mm. & 1927 & & & & $6 \cdot 6$ & $68 \cdot 4$ & $2 \cdot 1$ & - & - & - & - & - & - \\
\hline & 1928 & - & - & $6 \cdot 6$ & $37 \cdot 2$ & & & & & & & & \\
\hline & 1929 & & & & $14^{\circ} 7$ & 14.4 & 126 & - & - & - & - & & \\
\hline Pleuronectes flesus & 1924 & & & & & - & - & - & & & & & \\
\hline Flounder & 1925 & & & & $1 \cdot 2$ & $2 \cdot 7$ & - & - & - & & & & \\
\hline 4-9 $\mathrm{mm}$. & 1926 & & & & $6 \cdot 0$ & $0 \cdot 6$ & - & - & - & - & & & \\
\hline & 1927 & & & & $\begin{array}{r}3 \cdot 9 \\
10 \cdot 8\end{array}$ & $0 \cdot 2$ & - & - & - & - & - & - & - \\
\hline & $\begin{array}{l}1928 \\
1929\end{array}$ & - & - & 0.3 & $\begin{array}{r}10 \cdot 8 \\
0.6\end{array}$ & - & - & - & - & - & & & \\
\hline Pleuronectes & 1924 & & & & & $21 \cdot 9$ & $21 \cdot 6$ & $4 \cdot 5$ & & & & & \\
\hline microcephalus & 1925 & & & & $8 \cdot 7$ & $24 \cdot 6$ & $3 \cdot 9$ & 0.9 & - & & & & \\
\hline Merrysole or & 1926 & & & & $3 \cdot 6$ & $27 \cdot 6$ & $13 \cdot 2$ & $1 \cdot 2$ & - & - & & & \\
\hline Lemon Dab & 1927 & & & & $1 \cdot 5$ & $6 \cdot 0$ & $0 \cdot 6$ & - & - & - & - & - & - \\
\hline $4-13 \mathrm{~mm}$ & 1928 & - & - & $0 \cdot 3$ & $17 \cdot 7$ & & & & & & & & \\
\hline & 1929 & & & & - & $0 \cdot 6$ & $2 \cdot 7$ & 0.9 & - & - & - & & \\
\hline Solea vulgaris & 1924 & & & & & - & - & - & & & & & \\
\hline Common Sole & 1925 & & & & $2 \cdot 4$ & $1 \cdot 2$ & - & - & - & & & & \\
\hline 4-9 $\mathrm{mm}$ & 1926 & & & & 6.0 & 1.8 & $0 \cdot 2$ & - & - & - & & & \\
\hline & 1927 & & & & $6 \cdot 3$ & $1 \cdot 2$ & - & - & - & - & - & - & - \\
\hline & 1928 & - & - & 0.3 & $3 \cdot 3$ & & & & & & & & \\
\hline & 1929 & & & & & $0 \cdot 6$ & - & - & - & - & - & & \\
\hline Solea variegata & 1924 & & & & & $20 \cdot 1$ & 111.0 & $6 \cdot 1$ & & & & & \\
\hline Thickback & 1925 & & & & $9 \cdot 9$ & $101+4$ & $6 \cdot 3$ & 0.9 & - & & & & \\
\hline 4-11 mm. & 1926 & & & & $5 \cdot 7$ & $49 \cdot 8$ & $25 \cdot 5$ & $3 \cdot 9$ & - & - & & & \\
\hline & 1927 & & & 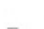 & $\begin{array}{r}2 \cdot 1 \\
10 \cdot 2\end{array}$ & 29.4 & 0.3 & $3 \cdot 6$ & - & - & - & - & - \\
\hline & $\begin{array}{l}1928 \\
1929\end{array}$ & - & & & 0.9 & 1.8 & $2 \cdot 4$ & $2 \cdot 7$ & - & - & - & & \\
\hline Solea lascaris & 1924 & & & & & - & - & - & & & & & \\
\hline Sand Sole & 1925 & & & & - & - & - & - & - & & & & \\
\hline & 1926 & & & & - & - & - & $\overline{-}$ & - & - & & & \\
\hline & 1927 & & & & - & - & - & 0.3 & - & - & - & - & - \\
\hline & 1928 & - & - & - & - & & & & & & & & \\
\hline & 1929 & & & & - & - & - & 0.4 & - & - & $0 \cdot 7$ & & \\
\hline
\end{tabular}




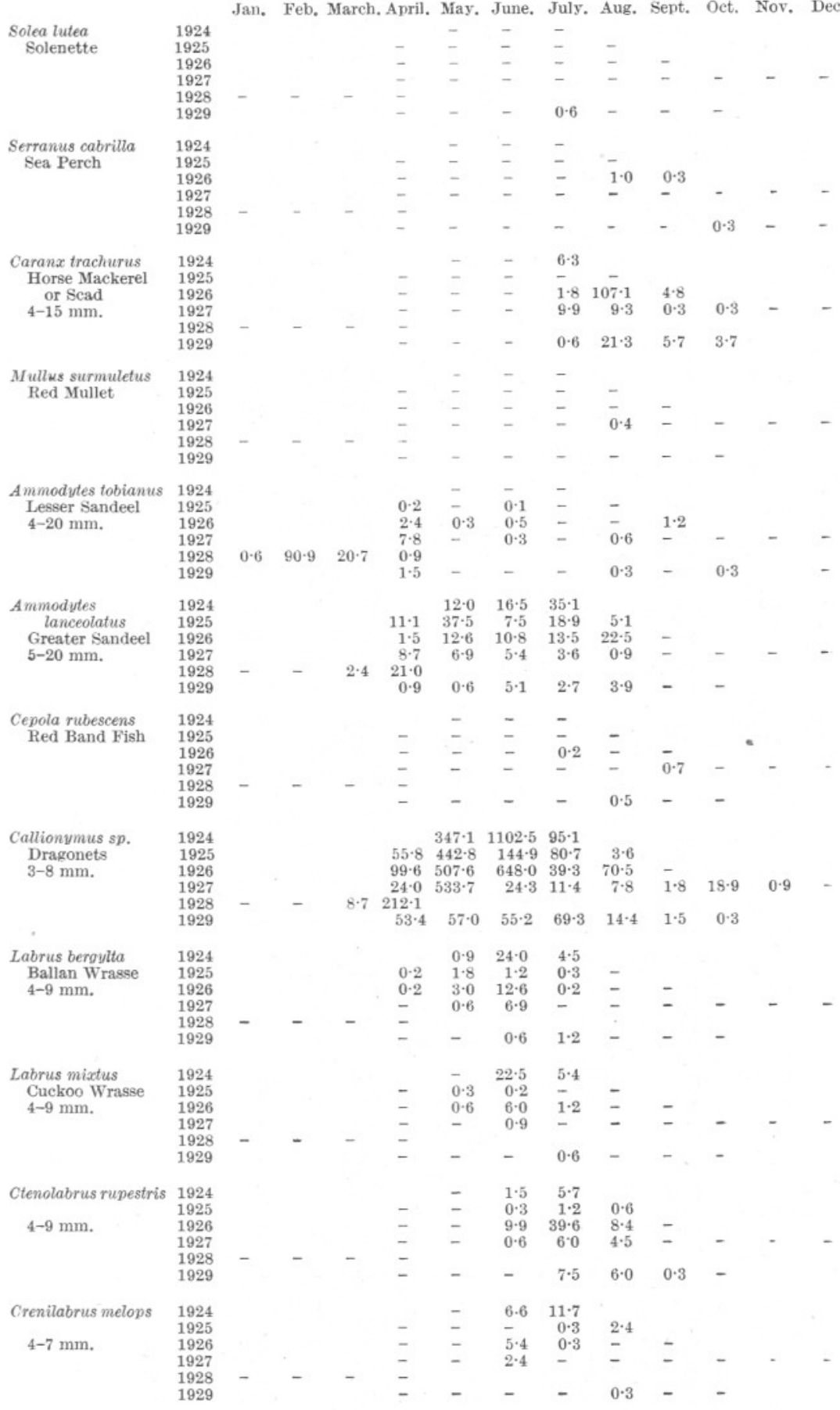




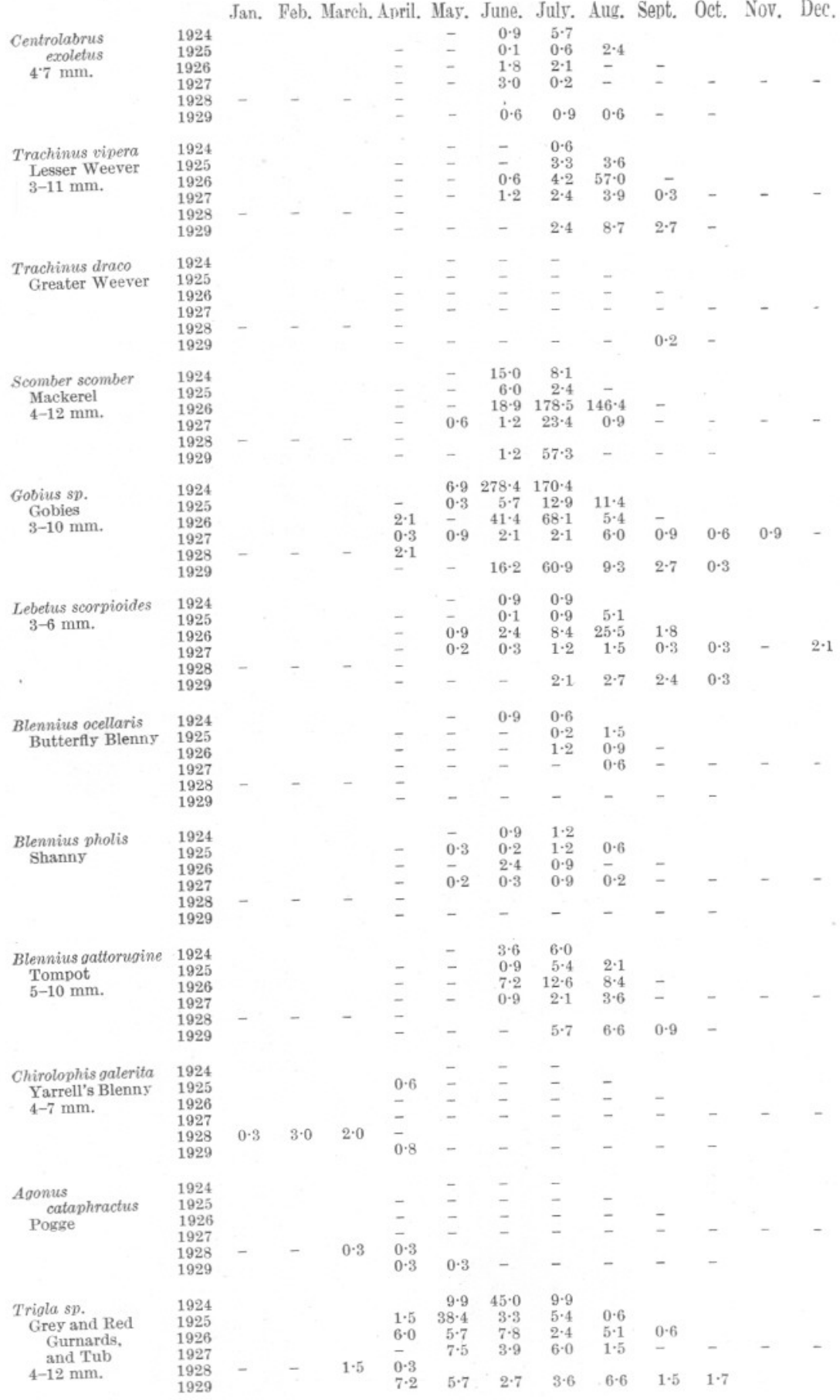


Jan. Feb. March, April. May. June. July. Aug. Sept. Oct. Nov. Dec.

\begin{tabular}{|c|c|c|c|c|c|c|c|c|c|c|}
\hline Cottus bubalis & 1924 & & & & & $0 \cdot 9$ & $1 \cdot 5$ & - & & \\
\hline Father Lasher & 1925 & & & & $0 \cdot 3$ & $0 \cdot 6$ & $0 \cdot 1$ & - & - & \\
\hline $5-8 \mathrm{~mm}$ & 1926 & & & & $1 \cdot 5$ & - & $0 \cdot 3$ & - & - & - \\
\hline & 1927 & & & & $1 \cdot 5$ & $0 \cdot 9$ & - & - & - & - \\
\hline & 1928 & - & - & $4 \cdot 5$ & $6 \cdot 0$ & & & & & \\
\hline & 1929 & & & & $3 \cdot 6$ & $0 \cdot 9$ & $0 \cdot 6$ & - & - & - \\
\hline Liparis montagui & 1924 & & & & & - & $3 \cdot 6$ & - & & \\
\hline Montague's Sucker & 1925 & & & & $0 \cdot 2$ & $1 \cdot 2$ & - & - & $1 \cdot 0$ & \\
\hline $7-10 \mathrm{~mm}$ & 1926 & & & & $0 \cdot 3$ & - & - & - & - & - \\
\hline & 1927 & & & & - & $3 \cdot 0$ & - & - & - & - \\
\hline & 1928 & - & - & - & $0 \cdot 8$ & & & & & \\
\hline & 1929 & & & & $0 \cdot 3$ & - & $0 \cdot 3$ & - & - & - \\
\hline Lepadogaster & 1924 & & & & & - & $7 \cdot 5$ & $3 \cdot 6$ & & \\
\hline bimaculatus & 1925 & & & & - & $0 \cdot 3$ & $0 \cdot 2$ & $1 \cdot 5$ & 0.9 & \\
\hline Doubly Spotted & 1926 & & & & - & $0 \cdot 3$ & $2 \cdot 4$ & $0 \cdot 6$ & $2 \cdot 1$ & - \\
\hline Sucker & 1927 & & & & $1 \cdot 5$ & - & - & - & $0 \cdot 2$ & - \\
\hline $5-10 \mathrm{~mm}$ & 1928 & - & - & - & - & & & & & \\
\hline & 1929 & & & & - & - & $2 \cdot 1$ & $2 \cdot 1$ & $2 \cdot 1$ & $0 \cdot 9$ \\
\hline Lophius piscatorius & 1924 & & & & & - & - & $1 \cdot 5$ & & \\
\hline Angler & 1925 & & & & - & - & - & - & - & \\
\hline 5-9 $\mathrm{mm}$ & 1926 & & & & $0 \cdot 2$ & - & $0 \cdot 6$ & 1.8 & - & - \\
\hline & 1927 & & & & - & - & $0 \cdot 3$ & $0 \cdot 3$ & $0 \cdot 2$ & - \\
\hline & 1928 & - & - & $0 \cdot 3$ & - & & & & & \\
\hline & 1929 & & & & - & - & $0 \cdot 6$ & 0.3 & - & - \\
\hline Belone vulgaris & 1924 & & & & & - & - & - & & \\
\hline Garfish & 1925 & & & & - & - & - & - & - & \\
\hline & 1926 & & & & - & - & - & - & - & - \\
\hline & 1927 & & & & - & - & - & - & - & - \\
\hline & 1928 & - & - & - & - & & & & & \\
\hline & 1929 & & & & - & - & - & - & - & 022 \\
\hline
\end{tabular}




\section{BIBLIOGRAPHY.}

1888. Cunningham, J. T. The Spawn of the Pilchard. Journ. Mar. Biol. Assoc., Old Series, No. 2. p. 247.

1891. _ On Some Larval Stages of Fishes (with Plates III and IV). Journ. Mar. Biol. Assoc., N.S., Vol. II, No. 1, p. 68.

1891. - The Reproduction and Growth of the Pilchard (with Plate X). Ibid., Vol. II, No. 2, p. 151.

1891. _ - The Egg and Larva of Callionymus lyra (with Plate V). Ibid., Vol. II, No. 2, p. 89.

1892. - Ichthyological Contributions. Ibid., Vol. II, No. 4, p. 325.

1892. Holt, E. W. L. Note on Scomber scomber Linn. (The Mackerel). Ibid., Vol. II, No. 4, p. 396.

Ibid., Vol. II, No. 4, p. 396.

1894. Cunningham, J. T. The Life-History of the Pilchard. Ibid., Vol III, No. 2, p. 148.

1894. - Young Stages of Zeugopterus punctatus. Ibid., Vol. III, No. 3, p. 202.

1895. - On a Specimen of Leptocephalus Morrisii. Ibid., Vol. IV, No. 1, p. 73 .

1895. - The Larva of the Eel. Ibid., Vol. III, No. 4, p. 278.

1897. Holt, E. W. L. Preliminary Notes on the Reproduction of Teleostean Fishes in the the South-Western District. Ibid., Vol. V, No. 1, p. 41.

1898. Notes on the Reproduction of Teleostean Fishes in the South-Western District. Ibid., Vol. V, No. 2, p. 107.

1898. Holt, E. W. L., and Scotт, S. D. A Record of the Teleostean Eggs and Larvæ observed at Plymouth in 1897. Ibid., Vol. V, No. 2, p. 156.

1898. Dunn, Matthias. On the Occurrence of Large Numbers of Larval Herrings at the Surface. Ibid., Vol. V, No. 2, p. 184.

1898. Holt, E. W. L., and Byrne, L. W. Notes on the Reproduction of Teleostean Fishes in the South-Western District. Ibid., Vol. V, No. 3, p. 333.

1900. Garstang, W. Preliminary Experiments on the Rearing of Seafish Larvæ. Ibid., Vol. VI, No. 1, p. 70. 
1903. Browne, F. Balfour. Report on the Eggs and Larvæ of Teleostean Fishes observed at Plymouth in the Spring of 1902. Ibid., Vol. VI, No. 4, p. 598.

1909. Sснмidt, J. The Distribution of the Pelagic Fry and the Spawning Regions of the Gadoids in the North Atlantic from Iceland to Spain. Conseil Perm. Internat. pour l'Explor. de la Mer. Rapp. et Proc. Verb., Vol. X, Special Part, No. 4.

1910. Hefford, A. E. Notes on Teleostean Ova and Larvæ. Journ. Mar. Biol. Assoc., N.S., Vol. IX, No. 1.

1914. Clark, R. S. General Report on the Larval and Post-Larval Teleosteans in Plymouth Waters. Ibid., Vol. X, No. 2, p. 327.

1917. Allen, E. J. Post-Larval Teleosteans Collected near Plymouth during the Summer of 1914. Ibid., Vol. XI, No. 2.

1918. Lebour, M. V. The Food of Post-Larval Fish. Ibid., Vol. XI, No. 4, p. 433.

1919. — The Food of Post-Larval Fish, No. II (1918). Ibid., Vol. XII, No. 1, p. 22.

1919. The Young of the Gobiidæ from the Neighbourhood of Plymouth. Ibid., Vol. XII, No. 1, p. 48.

1919. _ _ Feeding Habits of Some Young Fish. Ibid., Vol. XII, No. 1, p. 9.

1919. - Further Notes on the Young Gobiidæ from the Neighbourhood of Plymouth. Ibid., Vol. XII, No. 1, p. 146.

1920. Clark, R. S. The Pelagic Young and Early Bottom Stages of Teleosteans. Ibid., Vol. XII, No. 2, p. 159.

1920. Ford, E. The Post-Larval Stages of Ammodytes Species Captured during the Cruises of s.s. Oithona in Plymouth Waters in the Year 1919. Ibid., Vol. XII, No. 2, p. 241.

1920. - Note on a Leptocephalus Stage of the Conger. Ibid., Vol. XII, No. 2, p. 249.

1920. Lebour, M. V. The Eggs of Gobius minutus, pictus and microps. Ibid., Vol. XII, No. 2, p. 253.

1920. — The Food of Young Fish (1919). Ibid., Vol. XII, No. 2, p. 261.

1921. — The Larval and Post-Larval Stages of the Pilchard, Sprat and Herring from Plymouth District. Ibid., Vol. XII, No. 3, p. 427 . 
1921. — The Food of Young Clupeoids. Ibid., Vol. XII, No. 3, p. 458.

1922. Ford, E. On the Young Stages of Blennius ocellaris L., Blennius pholis L., and Blennius gattorugine L. Ibid., Vol. XII, No. 4, p. 688.

1922. - On the Post-Larvæ of the Wrasses occurring near Plymouth. Ibid., Vol. XII, No. 4, p. 692.

1924. Lebour, M. V. The Food of Young Herring. Ibid., Vol. XIII, No. 2, p. 325 .

1925. - Young Anglers in Captivity and Some of their Enemies. A Study in a Plunger Jar. Ibid., Vol. XIII, No. 3, p. 721.

1926. Russeld, F. S. The Vertical Distribution of Marine Macroplankton, II. The Pelagic Young of Teleostean Fishes in the Daytime in the Plymouth Area, with a Note on the Eggs of certain Species. Ibid., Vol. XIV, No. 1, p. 101.

1926. — The Vertical Distribution of Marine Macroplankton, III. Diurnal Observations on the Pelagic Young of Teleostean Fishes in the Plymouth Frea. Ibid., Vol. XIV, No. 2, p. 387.

1927. Lebour, M. V. The Eggs and Newly Hatched Young of the Common Blennies from the Plymouth Neighbourhood. Ibid., Vol. XIV, No. 3, p. 647.

1928. Russell, F. S. The Vertical Distribution of Marine Macroplankton, VIII. Further Observations on the Diurnal Behaviour of the Pelagic Young of Teleostean Fishes in the Plymouth Area. Ibid., Vol. XV, No. 3, p. 829.

1930. _ The Vertical Distribution of Marine Macroplankton, IX. The Pelagic Young of Teleostean Fishes in the Plymouth Area. Ibid., Vol. XVI, No. 2, p. 639. 